\title{
Distributed Scheduling Scheme for Video Streaming over Multi-Channel Multi-Radio Multi-Hop Wireless Networks
}

\author{
Liang Zhou, Xinbing Wang, Wei Tu, Gabriel-Miro Muntean, and Benoit Geller
}

\begin{abstract}
An important issue of supporting multi-user video streaming over wireless networks is how to optimize the systematic scheduling by intelligently utilizing the available network resources while, at the same time, to meet each video's Quality of Service $(\mathrm{QoS})$ requirement. In this work, we study the problem of video streaming over multi-channel multi-radio multihop wireless networks, and develop fully distributed scheduling schemes with the goals of minimizing the video distortion and achieving certain fairness. We first construct a general distortion model according to the network's transmission mechanism, as well as the rate distortion characteristics of the video. Then, we formulate the scheduling as a convex optimization problem, and propose a distributed solution by jointly considering channel assignment, rate allocation, and routing. Specifically, each stream strikes a balance between the selfish motivation of minimizing video distortion and the global performance of minimizing network congestions. Furthermore, we extend the proposed scheduling scheme by addressing the fairness problem. Unlike prior works that target at users' bandwidth or demand fairness, we propose a media-aware distortion-fairness strategy which is aware of the characteristics of video frames and ensures maxmin distortion-fairness sharing among multiple video streams. We provide extensive simulation results which demonstrate the effectiveness of our proposed schemes.
\end{abstract}

Index Terms-multi-channel multi-radio; video transmission; distributed scheduling; QoS; fairness.

\section{INTRODUCTION}

W ITH the motivation of improving the performance of multi-hop wireless networks, in the last few years great attention has been devoted to the networks where each node is equipped with multiple radio interfaces and can operate on multiple different channels [1]-[8]. The new degree of freedom has been proven to potentially allow for increased capacity with respect to single-channel single-interface networks [3], [4]. This is motivated by current WLAN standards where the entire frequency band is divided into multiple

Manuscript received 1 March 2009; revised 20 October 2009. Part of this paper is presented at IEEE Globecom 2009.

L. Zhou is both with the Lab. UEI, ENSTA-ParisTech, Paris, France and the Electronic Engineering Department, Shanghai Jiao Tong University, Shanghai, China (e-mail: liang.zhou@ieee.org).

$\mathrm{X}$. Wang is with the Electronic Engineering Department, Shanghai Jiao Tong University, Shanghai, China (e-mail: xwang8@sjtu.edu.cn).

W. Tu is with the Nokia Siemens Networks, Shanghai, China (e-mail: wei.tu@nsn.com).

G.-M. Muntean is with the Network Innovations Centre, Dublin City University, Dublin, Ireland (e-mail: munteang@eeng.dcu.ie).

B. Geller is with the Lab. UEI, ENSTA-ParisTech, Paris, France (e-mail: benoit.geller@ensta.fr).

Digital Object Identifier 10.1109/JSAC.2010.100412. channels, and each radio can only access one channel at a time. Therefore, if each network node has multiple radio interfaces, it can then utilize a larger amount of bandwidth, and hence achieve a higher system capacity [2], [3].

Such an improved bandwidth and capacity network poses a bright application foreground for large data video communications. However, there are huge and different kinds of videos streaming from different users which may influence each other and thus, it is essential to enforce a scheduling policy designed for suitable video metrics and efficient network utilization, preferably in a distributed manner. Indeed, the problem of video scheduling over multi-channel multi-radio networks is, compared to traditional data communications in wireless multi-hop networks, further complicated by the heterogeneity in both the network conditions and application contents, including i) channel-assignment: how to assign the channels that each link should be operated on? ii) rate allocation: how to allocate the appropriate rate to the given channels and links? iii) routing: how to select the potential channels and links that minimize total video distortion? and iv) fairness: how to provide a distortion-fairness for multiuser streaming different video clips concurrently? These four problems interact with each other, and thus form a challenging cross-layer control problem across the MAC layer and the application layer.

In this work, our objective is to propose a distributed video scheduling scheme in multi-channel multi-radio networks so as to minimize the total video's distortion and achieve a certain fairness. We first identify an objective function that balances the requests of the users and network operators, and then study how to construct a stable, dynamic and fair framework that optimizes for this objective. For ease of exposition, in the rest of the paper to avoid the confusion, we will use of the term "scheduling" to refer to the combined operation of channel assignment, rate allocation and routing. Although some scheduling protocols can be obtained via extending the current algorithms in [1], [2], [5] that are known to achieve the maximum system capacity or throughput for multi-channel multi-radio networks. However, these works completely ignore the transmission content. In addition, these works target at elastic communications, where users do not have stringent deadline constraints. Therefore, due to the characteristics of video content and the deadline requirement of video applications, these solutions may not be optimal for delivering multiuser, delay-constrained video applications. This point will be 
validated by the simulation results presented in Section IV of this paper.

Recently, many scheduling schemes have been proposed for video streaming over wireless multi-hop networks ( [12] and [13] provide a good overview). We summarize our contributions and the differences between our work and previous related works in the following.

- We provide a novel distributed video scheduling scheme in the context of multi-channel multi-radio multi-hop wireless networks. The support for multi-user video streams in this network requires appropriate joint channel assignment, rate control and multi-path routing measure, ascertaining the reasonable routes for transmitting each stream and the rate of the video to be delivered over the chosen routes. Different from previous works on video scheduling in single-channel multihop wireless networks [9], [12], [14] or multiple wireless networks [10], [11] in which channel assignment is not a concern, we consider the scheduling problem in the newly emerged networks and propose an efficient assignment algorithm. Moreover, unlike conventional works that consider routing for data traffic over wireless networks [6], [8], [9], we take into account the specific video characteristics in the routing and rate control scheme. Network congestion is considered in the channel assignment, rate allocation and routing metric, to meet the stringent delay requirement for video transmission. In addition, each video's rate-distortion characteristic is also taken into account in the joint routing and rate control procedure to provide multiple streams with various video contents. To the best of our knowledge, this work is the first one to deal with the video scheduling problem in the newly multi-channel networks.

- We extend the scheduling scheme by proposing a strategy of media-aware distortion-fairness, which is aware of the characteristics of video contents and ensures max-min distortion-fairness sharing among video streams. Every time when we talk about scheduling, fairness must be incorporated, as otherwise we will end up with a serious unfairness on network resource allocation, which has been shown by previous researches [15], [16]. There are existing works on max-min QoS fair sharing, such as [5], [15], [16], [25], [26]. These algorithms employ an explicit utility function of stream rate, but the video content is not taken into consideration. The work in [5] is not specifically developed for video and it does not take into account the special characteristics of application. What is more, the distributed algorithms in [15], [16], [25], [26] may need lots of compute complexity and their performances may vary dramatically such that the video quality fluctuates and the perceptual quality may be poor. It is worth noting that our method is also different from [15], [16]. Specifically, we do not employ any explicit utility function, but instead we use the importance of every frame which can be easily and explicitly calculated using the method in [23]. Furthermore, our scheme is content awareness and is operated over both links and sources, so our scheme belongs to perstream performance guarantee, which is also different from IntServ [17] architecture that offers application performance guarantees. In addition, our work has fundamental differences with DiffServ [18] which manages resources with the granularity of traffic classes.
The rest of the paper is organized as follows. Section II introduces the video distortion model, and formulates the scheduling as a convex optimization problem. In Section III, we propose a distributed minimum distortion scheduling scheme for video streaming over multi-channel multi-radio networks. Then, some simulation results and comparisons are provided for the corresponding scheme in Section IV. We extend the scheduling scheme by addressing the fairness problem, and provide a media-aware distortion-fairness strategy in Section V. Section VI concludes the paper and points to future work.

\section{PROBlem Formulation}

For the distortion of wireless video transmission, we employ an additive model to capture the total video distortion as [10], [21], [22], and the overall distortion $D_{\text {all }}$ can be obtained by:

$$
D_{\text {all }}=D_{\text {comp }}+D_{\text {loss }},
$$

where the distortion introduced by source compression is denoted by $D_{\text {comp }}$, and the additional distortion caused by packet loss is denoted by $D_{\text {loss }}$. According to [21], $D_{\text {comp }}$ can be approximated by:

$$
D_{\text {comp }}=\frac{\theta}{R-R^{0}}+D^{0},
$$

where $R$ is the rate of the video stream, $\theta, R^{0}$ and $D^{0}$ are the parameters of the distortion model which depend on the encoded video sequence as well as on the encoding structure. Likewise, $D_{c o m p}$ can be modeled by a linear model related to the packet loss rate $P_{\text {loss }}$ :

$$
D_{\text {loss }}=\alpha P_{\text {loss }},
$$

where $\alpha$ depends on parameters related to the compressed video sequence [21]. In a bandwidth-limited network, this combined loss rate can be further modeled based on the M/G/1 queuing model. In this case, the delay distribution of packets over a multi-hop network is exponential [9], [22]:

$$
\operatorname{Pr}\{\text { Delay }>T\}=e^{-\lambda T},
$$

where $\operatorname{Pr}\{\cdot\}$ denotes probability, $T$ reflects the delay constraint and $\lambda$ is the arriving rate which is determined by the average delay:

$$
\lambda=\frac{1}{E\{\text { Delay }\}} .
$$

In what follows, we study $E\{$ Delay $\}$ in the context of a specific wireless network.

Consider a multi-channel multi-radio wireless network with $\mathbf{N}=\{1, \ldots n \ldots, N\}$ nodes, $\mathbf{L}=\{1, \ldots l \ldots, L\}$ links, $N_{f}$ nonoverlapping frequency channels and each node $n \in \mathbf{N}$ is equipped with $N_{n}$ network interfaces. The basic network model is illustrated in Fig. 1. In order to take into account possible channel diversity, we denote $r_{l}^{c}$ as the rate at link $l \in \mathbf{L}$ can transfer data on channel $c$, provided that there are no interfering links transmitting on channel $c$ at the same time. Besides, there are $\mathbf{S}=\{1, \ldots s \ldots, S\}$ users in the system, and each user $s \in \mathbf{S}$ is associated with a source node and a destination node. The traffic from each user may be routed over multiple alternate paths. Let $\left[M_{s j}^{l}\right]$ denote the routing 


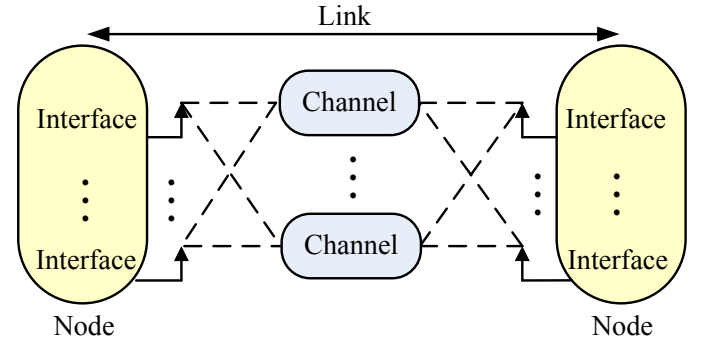

Fig. 1. Basic network model.

matrix, where $M_{s j}^{l}=1$ if path $j$ of user $s$ employs link $l$, $M_{s j}^{l}=0$, otherwise. Let $N(s)$ denote the number of alternate paths for user $s$, and $F_{s j}$ the fraction of traffic from user $s$ that is routed to path $j$. Furthermore, let $\mathbf{Q}=\left[Q^{c}\right]$ denote the outcome matrix of the routing scheme, where $Q^{c}$ is the set of non-interfering links that are chosen to transmit data in channel $c$. We denote Link Balance Ratio (LBR) [20] $u_{l}$ as the fraction of link input $r_{l}^{\text {in }}$ and link output $r_{l}^{\text {out }}$ for link $l$ :

$$
u_{l}=r_{l}^{\text {in }} / r_{l}^{\text {out }}
$$

where

$$
r_{l}^{i n}=\sum_{s=1}^{S} \sum_{j=1}^{N(s)} M_{s j}^{l} F_{s j} R_{s}
$$

and

$$
r_{l}^{\text {out }}=\sum_{c: l \in Q^{c}} r_{l}^{c},
$$

$R_{s}$ in (7) represents the video rate of user $s$.

Considering the interference relationship, for each link $l$, it is assumed that there is a set $\mathbf{I}_{l}$ of links that interfere with $l$. That is, if link $l$ and another link in $\mathbf{I}_{l}$ are transmitting on the same channel at the same time, neither of the links can transfer data, which is similar to the CSMA/CA mechanism used in 802.11 networks [20], [29]. We assume that each radio can only tune to one channel at any given time and switch channels dynamically as in [2], [6]. Therefore, for link $l$ to successfully communicate on channel $c$, both the sending and receiving nodes must tune one radio to channel $c$. In this case, the total $L B R$ in $\mathbf{I}_{l}$ can be defined as:

$$
u_{\mathbf{I}_{l}}=\sum_{l^{\prime} \in \mathbf{I}_{l}} u_{l^{\prime}}
$$

Congestion over each wireless link is measured as the average delay for all packets traversing that link. Following the classic M/G/1 queuing model, the average packet delay over a link is inversely proportional to the Potential Transmission Ability (PTA) [24]. Motivated by [19], we can set PTA of link $l$ as:

$$
P T A_{l}=r_{l}^{\text {out }} /\left(u_{\mathbf{I}_{l}}-\gamma\right),
$$

where $\gamma>1$ is an over-provisioning factor. Therefore, we can model the average packet delay for path $j$ of user $s^{1}$ :

$$
E\{\text { Delay }\}=\sum_{l=1}^{L} \frac{\left(u_{\mathbf{I}_{l}}-\gamma\right)}{r_{l}^{\text {out }}} \cdot \omega \cdot M_{s j}^{l},
$$

\footnotetext{
${ }^{1}$ In practice, congestion may be a more complicated function of rate as predicted by $\mathrm{M} / \mathrm{G} / 1$ model. However, this expression can be viewed as an approximation of the average link delay, capturing the non-linear increase of delay with total channel time utilization.
}

where $\omega$ is the average packet size. Therefore,

$$
\begin{aligned}
\operatorname{Pr}\{\text { Delay }>T\} & =e^{-\lambda T}=\exp \left\{-\frac{T}{E\{\text { delay }\}}\right\} \\
& =\exp \left\{-\frac{T}{\sum_{l=1}^{L} \frac{\left(u_{\mathbf{I}_{l}}-\gamma\right) \cdot \omega \cdot M_{s j}^{l}}{r_{l}^{\text {out }}}}\right\} .
\end{aligned}
$$

Taking into account the average packet loss rate $P_{B}$ due to transmission errors, the total packet loss rate for path $j$ of user $s$ is then:

$$
P_{\text {loss }}=P_{B}+\left(1-P_{B}\right) \operatorname{Pr}\{\text { Delay }>T\}
$$

The total distortion for path $j$ of user $s$ from packet loss can be expressed as

$$
D_{\text {loss }}=\alpha P_{\text {loss }}=\alpha\left(P_{B}+\left(1-P_{B}\right) \exp \left\{-\frac{T}{\sum_{l=1}^{L} \frac{\left(u_{\mathbf{I}_{l}}-\gamma\right) \cdot \omega \cdot M_{s j}^{l}}{r_{l}^{\text {out }}}}\right\}\right)
$$

Based on the previous discussions, we seek a joint optimal scheduling outcome $\mathcal{M}$ to achieve the overall minimum video distortion:

$$
\min _{\mathcal{M}}\left\{D_{\text {all }}=\sum_{s=1}^{S} \sum_{j=1}^{N(s)}\left(\frac{\theta_{s}}{R_{s}-R_{s}^{0}}+D_{s}^{0}+D_{\text {loss }}\right)\right\}
$$

subject to

$$
\begin{gathered}
r_{l}^{\text {out }}=\sum_{c: l \in Q^{c}} r_{l}^{c} \leq \sum_{s=1}^{S} \sum_{j=1}^{N(s)} M_{s j}^{l} F_{s j} R_{s}=r_{l}^{\text {in }}, \\
N(s) \geq 1, F_{s j} \geq 0, \sum_{j=1}^{N(s)} F_{s j}=1, \\
R_{s} \geq 0, n \geq 1, r_{l}^{c} \geq 0,
\end{gathered}
$$

where $\theta_{s}, R_{s}^{0}$ and $D_{s}^{0}$ in (15) are the corresponding parameters for user $s \in \mathbf{S}$. Intuitively, the reconstructed video quality is affected by the user's source rate $R_{s}$, the channel rate $r_{l}^{c}$, and the routing information $\left[M_{s j}^{l}\right]$. As mentioned before, this scheduling problem is implicitly coupled with a channel assignment, a rate allocation problem and a multi-path routing problem. In the next section, we propose a distributed algorithm where each source, each link and each channel jointly solve this scheduling problem through efficient cooperation.

\section{Distributed Minimum-Distortion Scheduling SCHEME}

The optimization problem defined in (15)-(18) involves several network layers, and we propose a 3-step method to resolve this problem. First, we propose a channel assignment algorithm by using convex programming formulation to simplify the goal function, and the constraints placed by nodes, links, and channels to "fix" the rate allocation and routing. We aim st constructing the mapping relationship between the channel assignment and video distortion. Second, we develop a joint rate allocation and multi-path routing algorithm to achieve the trade-off between the coding distortion and network congestion. Third, we provide the exact operation steps for the 


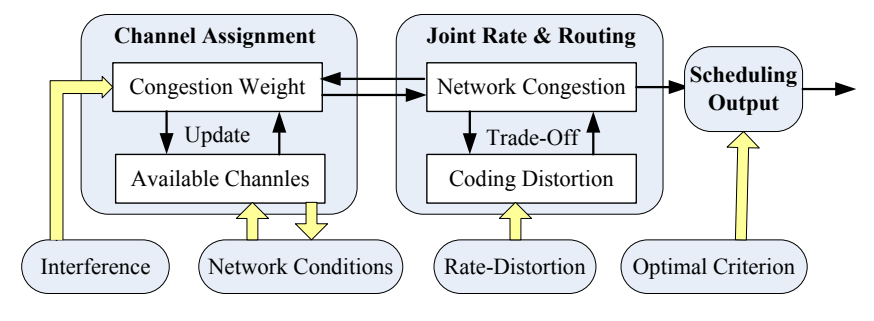

Fig. 2. Block diagram of distributed scheduling scheme.

distributed minimum-distortion scheduling (DMDS) solution based on channel assignment, rate allocation and multi-path routing computed in the previous two steps. The system diagram of the distributed scheduling scheme is displayed in Fig. 2.

\section{A. Channel Assignment}

In the processing of channel assignment, we focus on every node to select "optimal channels" to achieve minimum video distortion. However, it is difficult to define the "minimum video distortion" in the process of channel assignment. Hence, we map the index of "minimum video distortion" to "optimal network congestion" 2 . Specifically, we assume a linear programming (19) method to obtain approximate solutions of the optimal channel assignment. In this formulation, we define $\left(r_{l}^{\text {in }}-r_{l}^{\text {out }}\right)$ as the factor of network congestion, and the corresponding constraints remain identical to (17) and (18).

$$
\min \sum_{l \in \mathbf{L}}\left(r_{l}^{\text {in }}-r_{l}^{\text {out }}\right)
$$

The sketch of the proposed Channel Assignment Algorithm (CAA) is provided in Table I. The optimal channel assignment solution $\mathbf{A}$ is computed by solving the linear programming equation (19). During the execution of CAA, if there is a link $l$ from node $n$ to node $m$, then $N_{c}$ records the number of the common channels in nodes $n$ and $m$, while $N_{n}$ and $N_{m}$ record the number of available network interfaces of node $n$ and $m$, respectively. $N_{f c}$ represents the number of the required channels which is determined by the corresponding allocated rate in the selected link $l$ and the number of available network interfaces. Note that the congestion weight of channel $c$ in link $l$ is $u_{l}^{c}$, which can be defined as:

$$
u_{l}^{c}=u_{l} / r_{l}^{c} \text {. }
$$

When assigning a channel $c$ to link $l, r_{l}^{c}$ amount of stream is allocated, and can be updated during the execution of the $\mathrm{CAA}^{3}$.

Remark: The purpose of choosing a channel with the smallest congestion weight is to make the channels assigned to spatially close nodes as different as possible [5]. Note that in the replacement procedure of ChannelAssignment (please see the steps 12, 17, 21, and 35 in Table I), we always use the selected channel to replace the channel with the largest congestion weight. In the worst case, the algorithm will eventually stop after passing through all $N$ nodes.

\footnotetext{
${ }^{2}$ As stated previously, the stream rate is fixed in the process of channel assignment, so $D_{\text {comp }}$ is not changed in this process.

${ }^{3} r_{l}^{c}$ is initialized with the capacity of channel $c$.
}

TABLE I

Channel Assignment Algorithm (CAA)

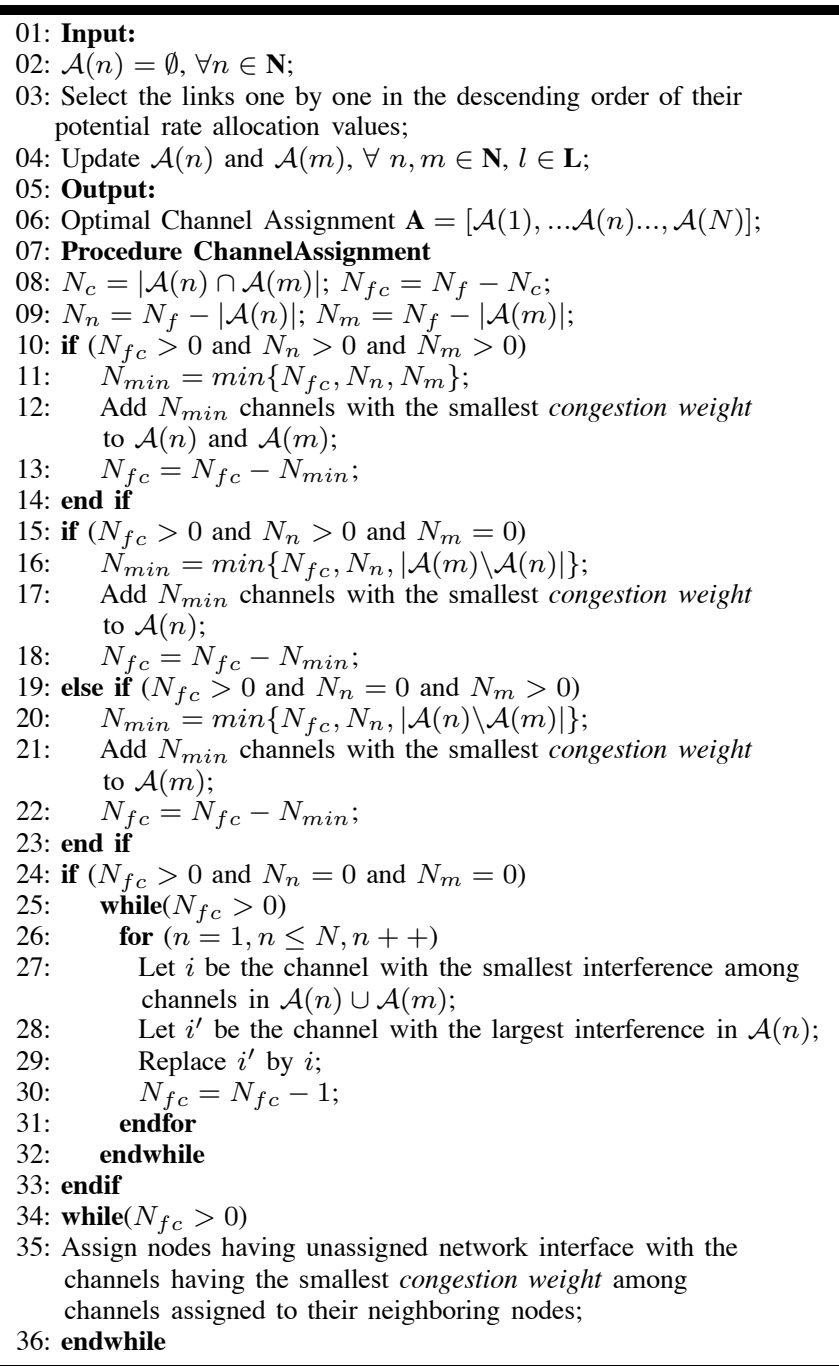

\section{B. Joint Rate Allocation and Multi-Path Routing}

Here, we employ multi-path routing with the goal of finding multiple potential paths to minimize the total system congestion induced by each user. Motivated by [22], we consider dividing the total rate increment of each video stream $\triangle R_{s}$ into $K(K \geq 1)$ small increments (corresponding to $N(s)$ paths described in Section II) such that $\triangle R_{s}=\sum_{k=1}^{K} \triangle R_{s}^{k}$. Assuming that $(k-1)$ of the $K$ paths and their increments are already known, path $P_{s}^{k}$ and its increment can be determined to achieve the minimal congestion. The average delay on each link is proportional to $1 / P T A_{l}^{\prime}$ using the $\mathrm{M} / \mathrm{G} / 1$ queuing model:

$$
P T A_{l}^{\prime}=r_{l}^{o u t^{\prime}} /\left(u_{\mathbf{I}_{l}}-\gamma\right),
$$

where $r_{l}^{\text {out }}=r_{l}^{\text {out }}+\sum_{k^{\prime}=1}^{k-1} \triangle R_{s}^{k^{\prime}}$ denotes the existing current traffic of link $l$ in $P_{s}^{k}$ plus the potential contributions from the other $k-1$ path rate increments. Therefore, we can choose another appropriate $P_{s}^{k}$ for the next increment $\triangle R_{s}^{k}$, such that

$$
\min _{\triangle R_{s}^{k}} \sum_{l \in \mathbf{L}} \frac{r_{l}^{\text {out }}+\triangle R_{s}^{k}}{P T A_{l}^{\prime}-\triangle R_{s}^{k}} .
$$


Actually, this is also equal to optimizing the increase congestion in the total network:

$$
\min _{\triangle R_{s}^{k}} \sum_{l \in \mathbf{L}}\left(\frac{r_{l}^{\text {out }}+\triangle R_{s}^{k}}{P T A_{l}^{\prime}-\triangle R_{s}^{k}}-\frac{r_{l}^{\text {out }}}{P T A_{l}^{\prime}}\right) \approx \min _{\triangle R_{s}^{k}} \sum_{l \in \mathbf{L}} \frac{\triangle R_{s}^{k}}{P T A_{l}^{\prime}} .
$$

The approximation holds when $\triangle R_{s}^{k}$ is small, which also restricts the traffic assignment for $\triangle R_{s}^{k}$ to be assigned to other paths other than $P_{s}^{k}$. This results in a sub-optimal solution to (22), but since the increment is small, the degradation in performance is expected to be insignificant.

Therefore, the optimal allocation of increment $\triangle R_{s}^{k}$ can be realized by finding a path $P_{s}^{k}$ from source to destination minimizing the increase congestion in the network. Since only links in $P_{s}^{k}$ make a change, the optimization problem becomes:

$$
\min _{P_{s}^{k}} \sum_{l \in P_{s}^{k}} \frac{\triangle R_{s}^{k}}{P T A_{l}^{\prime}} .
$$

We now jointly consider routing and rate allocation problem by optimally allocating rate to each video stream among multiple paths. The necessary and sufficient conditions for the optimal solution to (15) are the allocated rate to each stream should meet the boundary condition exactly:

$$
\frac{d D_{\text {comp }}}{d R_{s}}+\frac{d D_{\text {loss }}}{d R_{s}}=0
$$

where $d D_{\text {comp }} / d R_{s}$ is derived from the video distortion model (15) as:

$$
\frac{d D_{c o m p}}{d R_{s}}=-\frac{\theta_{s}}{\left(R_{s}-R_{s}^{0}\right)^{2}} .
$$

Therefore, the distortion reduction caused by increasing encoding rate by $\triangle R_{s}^{k}$ is:

$$
-\triangle D_{\text {comp }}^{k} \approx \frac{\theta_{s}}{\left(R_{s}-R_{s}^{0}\right)^{2}} \triangle R_{s}^{k} .
$$

On the other hand, the slope of packet loss distortion increment $d D_{\text {loss }} / d R_{s}$ can be expressed as:

$$
\begin{aligned}
\frac{d D_{\text {loss }}}{d R_{s}} & \propto \alpha\left(1-P_{B}\right) \sum_{l \in \mathbf{L}} \frac{u_{\mathbf{I}_{l}}-\gamma}{r_{l}^{\text {out }}} \\
& \approx \alpha\left(1-P_{B}\right) \sum_{l \in \mathbf{L}} \frac{\sum_{l^{\prime} \in \mathbf{I}_{l}}\left(u_{l^{\prime}}-\gamma\right)}{r_{l}^{\text {out }}} \\
& \approx \alpha\left(1-P_{B}\right) \sum_{l \in \mathbf{L}} \frac{1}{P T A_{l}^{\prime}} .
\end{aligned}
$$

$r_{l}^{\text {out }}{ }^{\prime}$ is also the cross-traffic which includes contributions from current traffic $r_{l}^{\text {out }}$ and other video streams. Then, the resulting packet loss distortion increment $\triangle D_{\text {loss }}^{k}$ can be approximated as:

$$
\triangle D_{l o s s}^{k} \approx \alpha\left(1-P_{B}\right) \sum_{l \in \mathbf{L}} \frac{\triangle R_{s}^{k}}{P T A_{l}^{\prime}} .
$$

Note that (29) is almost the same as the optimization formulation in (24), and can be accumulated over the chosen links on one path.

Remark: Given the packet loss distortion increment $\triangle D_{\text {loss }}^{k}$ in (29) and the video compression distortion reduction $\triangle D_{\text {comp }}^{k}$ in (27), the source can make the rate allocation
TABLE II

THE DMDS SCHEME

$$
\text { At each time slot } t \text { : }
$$

- Source s: determine the optimal path distribution for each source

$$
\begin{aligned}
& \max _{F_{s}} \quad-\sum_{j}\left(F_{s j}\right)^{2}-\sum_{j} F_{s j} \sum_{l} M_{s j}^{l} q_{l}(t) \\
& \text { where } F_{s}=\left[F_{s 1} \ldots, F_{s j}, \ldots F_{s N s}\right], F_{s j} \geq 0, \\
& \text { and } q_{l}(t) \text { denotes the queue length for link } l \text { at time slot } t ; \\
& \text { Queue Length Update: } \\
& q_{l}(t+1)=\left[q_{l}(t)+\lambda_{q}(t)\left(\sum_{s} \sum_{j} M_{s j}^{l} F_{s j} R_{s}^{j}(t)-\sum_{c} r_{l}^{c}(t)\right)\right]^{+} \\
& \text {where }[x]^{+}=\max (x, 0), \text { and } \lambda_{q}(t) \text { is the step size. }
\end{aligned}
$$

- Link I: determine the optimal traffic in each link

$$
\min _{P_{s}^{k}} \sum_{l \in P_{s}^{k}} \frac{\triangle R_{s}^{k}(t)}{P T A_{l}^{\prime}}
$$

Rate Increment Update:

$$
\triangle R_{s}^{k}(t+1)=\left[\triangle R_{s}^{k}(t)+\lambda_{R}(t)\left(\sum_{k} \triangle D_{\text {loss }}^{k}-\triangle D_{\text {comp }}^{k}\right)\right]^{+}
$$

where $\lambda_{R}(t)$ is the step size.

- Channel c: determine the minimum congestion in each channel

$\min \sum_{l}\left(r_{l}^{\text {in }}-r_{l}^{\text {out }}\right)$

Congestion Weight Update:

$u_{l}^{c}(t+1)=u_{l}(t+1) / r_{l}^{c}(t+1)$.

decision by comparing these two quantities. The allocated rate will be increased by $\triangle R_{k}^{s}$ until $-\triangle D_{\text {comp }}^{k}>\triangle D_{\text {loss }}^{k}$. Therefore, the rate control algorithm can continue until it reaches the optimal rate that achieves a balance between the rate increment and network congestion [19].

\section{Distributed Scheduling Scheme}

Based on the given channel assignment, the joint rate allocation and routing algorithms, DMDS scheme is provided to present an optimal scheduling scheme for video transmission over multi-channel multi-radio multi-hop wireless networks. The main challenges in designing DMDS are how to select optimal channels, paths as well as allocated rates to ensure the performance is both stable and optimal. We illustrate the interplay between the source, link and channel in Table II. For DMDS, each channel computes the congestion weight to make the channels assigned to spatially close nodes as different as possible, each link calculates the rates to achieve a balance between the rate increment and network congestion, and each source determines the optimal path distribution to achieve minimum video distortion. Specifically, congestion weight message is fed back from the channels to the links to avoid network congestion, queue length message is from the links to the sources to prevent the source rates from exceeding the transmission ability, and rate allocation and routing message is from the sources to the links to achieve the optimal performance.

Specifically, the sources send all of the links with path discovery messages, which are relayed by the intermediate nodes on the control channel. At each intermediate node, the path discovery messages contain the information of congestion 


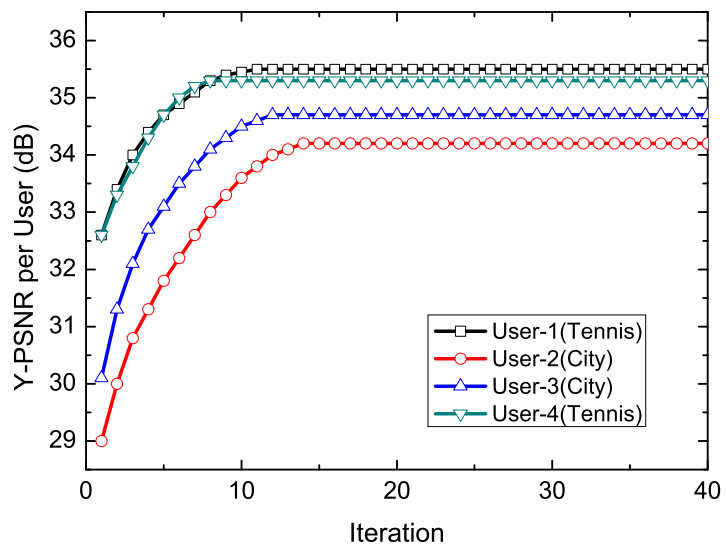

Fig. 3. Plots of PSNR versus time for the 1st frame of each video (step sizes: $\lambda_{q}=4 \times 10^{-5}, \lambda_{R}=2 \times 10^{-5}$ ).

weight and queue length related to every possible stream between the source and intermediate nodes. Then, the intermediate node extends the path as the source does. Upon reception of path discovery messages from the destinations, the sources determine the possible paths based on the feedback from the links, in form of queue length, rate increment and congestion weight. In particular, the source minimizes the total distortion while balancing the congestion of channels and links.

Remark: From Table II, it can be found that the computations at the sources are linear with the number of sources, while the computations at the links and channels do not grow with the number of sources. Specifically, determining the minimum congestion channel takes a constant time $O\left(N_{f}\right)$. The complexity of finding optimal traffic in each link is $O\left(N_{n} \cdot N\right)$, since each node $n \in \mathbf{N}$ is equipped with $N_{n}$ interfaces. The complexity caused by all the links is $O\left(L \cdot N_{f}+L \cdot N_{n} \cdot N\right)$. In addition, the complexity of multiple-path routing for each source $s \in \mathbf{S}$ is a constant $O(N(s))$. Therefore, for each source $s \in \mathbf{S}$, the computation complexity of all the users is $O(S \cdot N(s))$. Therefore, the total computation complexity is $O\left(L \cdot N_{f}+L \cdot N_{n} \cdot N+S \cdot N(s)\right)$.

Proposition 1: DMDS scheme converges to the joint global optimum $\mathcal{M}$ of (15) for sufficiently small queue length step size $\lambda_{q}$ and rate increment step size $\lambda_{R}$.

Proof: Please see Appendix.

\section{Simulation of the DMDS Scheme}

In this section, we conduct extensive simulations to study the performance of the proposed DMDS scheme in multichannel multi-radio multi-hop wireless networks. Concerning the unreliable control channels, we use retransmission mechanism to ensure that the information exchange between each node is available. In addition, we employ the mechanism introduced in [27] and [28] to implement reliable control channel and node synchronization, respectively. To simulate the video applications, two HD (High-Definition) sequences (City and Tennis) are used to represent video with dramatically different levels of motion activities. In terms of HD video, the sequence has spatial resolution of $1280 \times 720$ pixels, and the frame rate of 60 frames per second. Video stream is encoded using a fast implementation of H.264/AVC codec at various quantization step sizes, with GOP (Group Of Pictures) length of 25 and $I B B P$... structure similar to that often used in MPEG-2 bitstreams. Encoded video frames are segmented into packets with maximum size of 1500 bytes, and the transmission intervals of each packet in the entire GOP are spread out evenly, so as to avoid unnecessary queuing delay due to the large sizes of intra coded frames. In the following, we set $T=300 \mathrm{~ms}, P_{B}=1 \%$, and $\alpha=350$ unless otherwise specified.

To study the characteristics of the proposed DMDS scheme, we experiment with the setting of $S=4, N=10, L=15$, $N_{f}=2, N_{n}=2$. The simulation results are presented in Fig. 3. It can be observed from Fig. 3 that the curves follow an increasing concave trajectory, converging close to the optimum in less than 15 iterations. While the graphs in Fig. 3 are for one particular initial condition, we have done simulations for a variety of initial conditions to verify that the convergence time is independent of the initial conditions. In addition, it should be noted that, in all experiments, we start with an initial routing configuration (i.e. the earliest path known by the source) that splits the traffic evenly among the paths for each source-destination pair. For background stream, it is generated according to an on/off source model with exponential distribution of staying time, and average rates between 0 and $0.2 \cdot r_{l}^{i n}$ for each link.

To demonstrate the effectiveness of our proposed scheme, DMDS is benchmarked against other two popular scheduling schemes for multi-channel multi-radio wireless networks : i) Maximum Throughput Scheduling (MTS) introduced in [5], in which this scheme seeks for a feasible end-to-end rate allocation vector along with feasible channel assignment to achieve optimal throughput; ii) Provably-Efficient Maximal Scheduling (PEMS) introduced in [2], in which a distributed on-line algorithm is provided to achieve a provable fraction of the maximum system capacity. Fig. 4 shows the first 200 frames achieved by four users requesting two different video clips under the given network realization. From Fig. 4, we can see that compared to MTS and PEMS schemes, our proposed DMDS scheme has a considerable performance advantages. That is because the above competing schemes only consider the rate maximization or throughput optimum, while our scheme aims at minimum video distortion by jointly considering the characteristics of network and video. Note that some of the frame's PSNR values of MTS and PEMS may be higher than that of our proposed DMDS scheme, however without any significant performance improvement compared to the video quality of our proposal.

Then, we test the proposed DMDS scheme in a dynamic environment where users can join or leave the given network randomly. We start with 4 users (two are City and two are Tennis). At time $t=2 s$, we add 4 new users (two are City and two are Tennis), and at time $t=8 \mathrm{~s}$, we randomly remove 2 users (one is City and one is Tennis). Fig. 5 presents the average PSNR for original 4 users obtained by proposed DMDS scheme. From Fig. 5, an interesting observation can be found that our proposed DMDS scheme can achieve a 


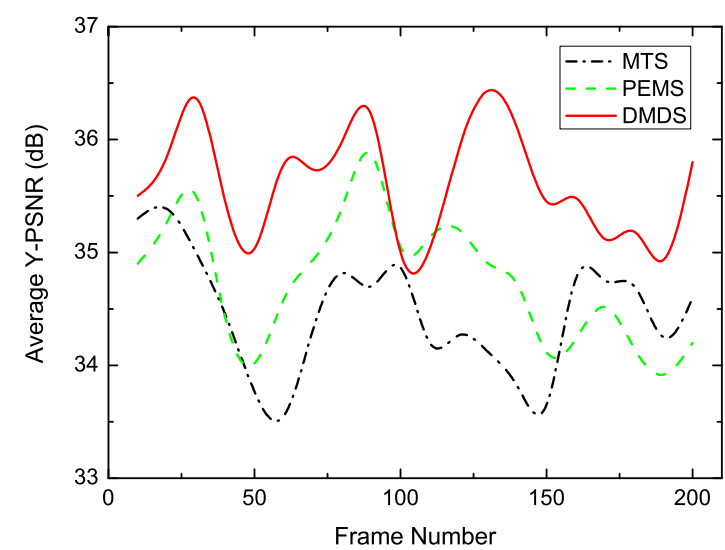

(a) Tennis (User-1 and User-4)

Fig. 4. Plots of average PSNR versus time for two different videos.

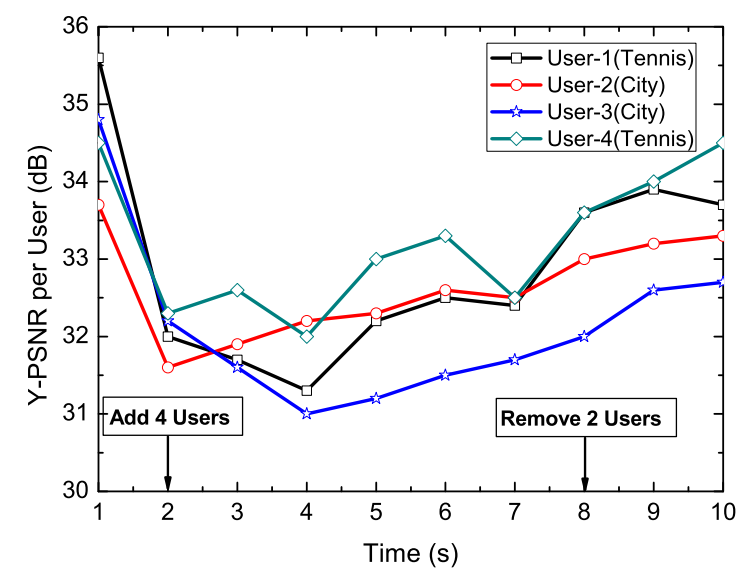

Fig. 5. Average performance per user in case users join/leave the network.

satisfying average performance in this dynamic environment, however, the performance differences between each frame and each video vary dramatically. Meaning that the distortionfairness property of DMDS is not so good. For example, the standard derivation of each frame for user- 1 is 1.29 $\mathrm{dB}$, and the average performance difference between user- 3 and user-4 is $1.20 \mathrm{~dB}$. In some cases, distortion-fairness is also an important issue for video transmission, especially for multi-user wireless environments. Given the above simulation results, a natural question arises: can we develop a distributed distortion-fairness scheduling algorithm that can take into account different video contents based on the proposed DMDS scheme? In the next section, we will study this problem.

\section{Extension: Media-Aware Distortion-Fairness DisTRIBUTED SCHEDULING SCHEME}

Nowadays, there exists lots of fairness strategies in terms of bandwidth sharing and demand satisfaction [5], [15]. However,

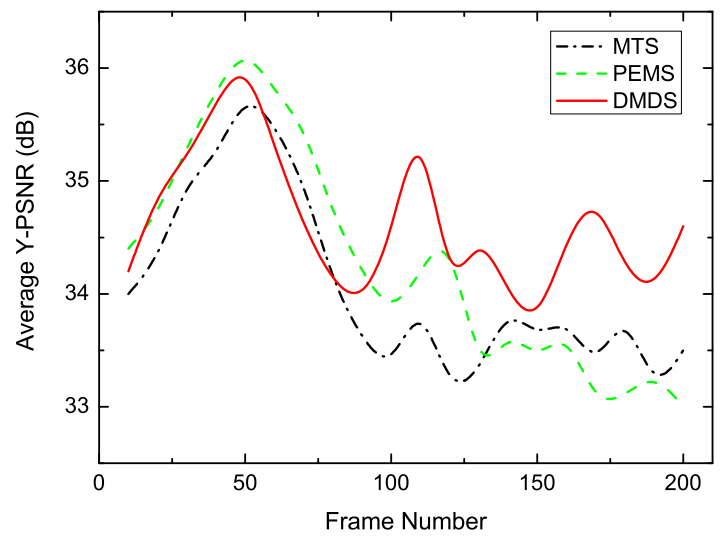

(b) City (User-2 and User-3)

users care about QoS rather than the bandwidth or demand, and the same QoS may need different bandwidth or demand according to different video contents. In this section, we propose a media-aware distortion-fairness strategy which is aware of the characteristics of video frames and ensures maxmin distortion fair sharing among video steams based on the proposed DMDS scheme.

\section{A. Media-Aware Distortion-Fairness Strategy}

The procedure of the Media-Aware Distortion-Fairness (MADF) strategy is as follows. Sources send messages indicating the importance of each frame to the links, then the sources and links cooperatively decide the optimal threshold of dropping frames for each user, where the frames with less importance than the threshold will be dropped [25]. The scheduling scheme and the distortion metrics in the application layer are the same as the proposed DMDS scheme. The resulting MADF strategy, in which the sources and links can cooperate with others such that the end-to-end distortion fairness as well as satisfying video quality are achieved among users. Here we define the importance of each frame based on the inter-frame motion as [23], while taking into account of the prediction structures of the frames. It is worth noting that each shared link decides a common distortion threshold of dropping frames for all users for the case of multiple users sharing links, therefore, each user can satisfy the distortion caused by the congestion. The same threshold of dropping frames makes each user experiences a max-min fairness distortion [25]. Fig. 6 illustrates the architecture of the MADF with dropping frame strategy that exchanges information between the sources and links.

The core procedure of MADF strategy is to find the optimal threshold of dropping frames for each user. First, each link finds an equal distortion level to its users that are not marked with "Flag" and each user sets its frame dropping level as the most stringent one over all the links on its path. Second, if the link is bottlenecked based on its users' updated dropping level, the link marks itself and the users mark it "Flag", and other 


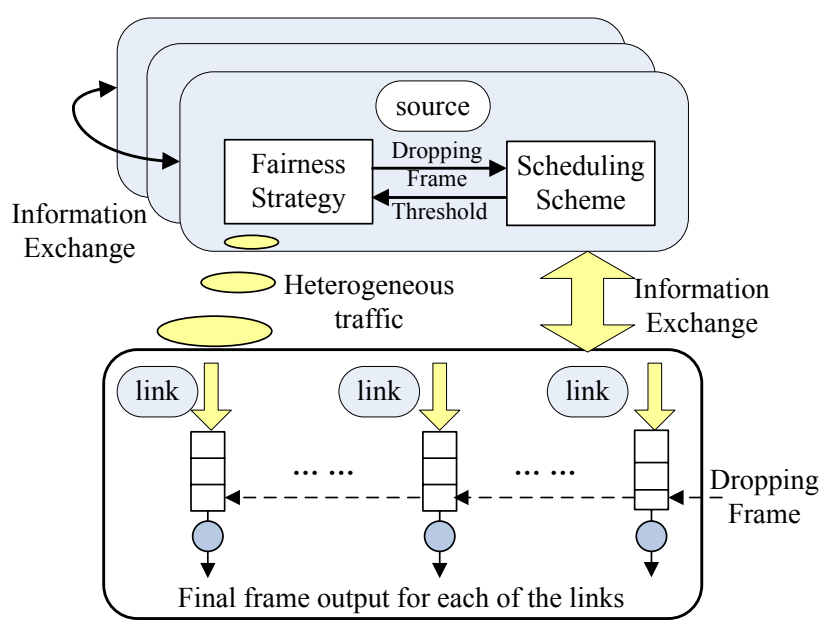

Fig. 6. Architecture of the MADF scheduling scheme with dropping frame strategy.

links drop corresponding frames of the users marked "Flag". Third, the iteration goes to another round. In this way, it is possible to make each user gets distortion as low as possible and fairly, and the distortion of the user who experiences most stringent bottleneck is minimized. Therefore, we can achieve the max-min distortion fairness share. The detail operation of MADF is presented in Table III.

Remark: The work in [25] is similar to our media-aware distortion-fairness strategy; however, the main focus of [25] is how to optimize the congested network, whereas our work aims at reducing the distortion unfairness in specific wireless setting. The authors of [5] develop a proportional fair endto-end rate allocation for multi-radio wireless mesh networks by introducing Demand Satisfaction Factor (DSF). The DSF of a session is defined as the ratio between the rate actually allocated to that session and its traffic demand. In fact, the algorithm in [5] just considers the demand-fair for each user, and does not consider the characteristics of video streaming. We refer the readers to the discussions in the Introduction part regarding the difference between our work and [5], [25] and the potential implications.

Proposition 2: The additional number of iterations induced by MADF is at most the number of bottlenecks, where the bottleneck is counted in the same network where all the streams are fully elastic without any upper bound.

Proof: In each iteration, there will be at least a link whose current threshold will not change in the next iteration, and such link is a link which would be fully utilized if the streams on it are fully elastic without any upper bound.

Next we consider the cost (penalty) function $D\left(z_{l}, z_{l}^{\prime}\right)$ when MADF is employed. (14) can be rewritten as

$$
D_{\text {loss }}=\alpha P_{\text {loss }}+\sum_{l \in Q^{c}} D\left(z_{l}, z_{l}^{\prime}\right) .
$$

From (30), each link will keep updating its state information, unless the performance difference of employing the proposed dropping frame algorithm becomes small. Hence, in the proposed MADF strategy, assume the difference between the current strategy $z_{s}$ and the previous strategy $z_{s}^{\prime}$ for user $s$
TABLE III

MADF STRATEGY

01: Input:

02: All links are set 'Flag=FALSE', and $z_{l}=\max I$;

03: All users are set 'Flag=FALSE', and $z_{s}=\max I$;

where $\max I$ is a given upper bound of the frame importance;

04: Output:

05: Optimal Media-Awareness Distortion-Fairness Scheduling;

06: Procedure FrameDrop

07: if (link $l$ 'Flag=FALSE')

08: The frame queuing in link $l$ whose importance level

below the threshold $z_{l}$ is dropped;

09: Recalculates the threshold $z_{l}^{\prime}$ for the update queue;

10: $\quad$ if $\left(z_{l} \neq z_{l}^{\prime}\right)$

11: $\quad$ Link $l$ is set 'Flag=TRUE';

12: User relevant to $l$ is set 'Flag=TRUE' and $z_{s}=\max \left\{z_{l}, z_{l}^{\prime}\right\}$;

13: endif

14: endif

15: if (user $s$ 'Flag=FALSE')

16: The frame dropping level is set $z_{s}=\max z_{l}$ over all the links on its path;

17: $\quad$ User $s$ is set 'Flag=TRUE';

18: else

19: User $s$ drops the frames whose importance is less than $z_{s}$;

20: endif

21: Procedure DistributedScheduling

22: The same to the DMDS scheduling presented in Table II;

is $e_{s}$, i.e., $e_{s}=\left|z_{s}-z_{s}^{\prime}\right|$. Let

$$
U_{s}^{\text {diff }}=e_{s} \times \max \left[z_{l}^{\prime} \sum_{j} M_{s j}^{l} q_{l}\right]
$$

be the distortion difference between the current strategy and the previous strategy. Note that, according to the proposed dropping frame algorithm, we choose the max operation in the distortion expression.

Claim 1: If $e_{s}$ satisfies the following condition:

$$
U_{s}^{\text {diff }} \leq \sum_{l \in Q^{c}} D\left(z_{l}, z_{l}^{\prime}\right)
$$

for all users $s \in \mathbf{S}$, the proposed scheduling scheme converges to a stable state.

Proof: Equation (32) can be derived as:

$$
\begin{aligned}
\sum_{l \in Q^{c}} D\left(z_{l}, z_{l}^{\prime}\right) & \geq \sum_{l \in Q^{c}}\left\{\left(z_{l}-z_{l}^{\prime}\right) \times \max \left[z_{l}^{\prime} \sum_{j} M_{s j}^{l} q_{l}\right]\right\} \\
& \Rightarrow z_{l}^{\prime} \times \max \left[z_{l}^{\prime} \sum_{j} M_{s j}^{l} q_{l}\right] \\
& \geq z_{l} \times \max \left[z_{l}^{\prime} \sum_{j} M_{s j}^{l} q_{l}\right]-D\left(z_{l}, z_{l}^{\prime}\right) \\
& \geq z_{s} \times \max \left[z_{l}^{\prime} \sum_{j} M_{s j}^{l} q_{l}\right]-D\left(z_{s}, z_{s}^{\prime}\right) \\
& \Rightarrow U_{s}^{\text {diff }} .
\end{aligned}
$$

The remaining proof follows [2, Proposition 2], so the strategy converges to a stable state.

Claim 2: If the penalty function $D\left(z_{l}, z_{l}^{\prime}\right)$ is a convex function of $z_{l}$, when the proposed dropping frame algorithm converges to a stable state, the distortion difference between the proposed MADF strategy and the DMDS scheme is not larger than $\sum_{l \in Q^{c}} D\left(z_{l}, z_{l}^{\prime}\right)$.

Proof: As long as the penalty function $D\left(z_{l}, z_{l}^{\prime}\right)$ is a convex function of $z_{l}$, the additional video distortion function is a 
TABLE IV

PERFORMANCE COMPARISON FOR DIFFERENT SCHEMES IN DIFFERENT SCENARIOS

\begin{tabular}{|c|c|c|c|c|c|c|c|c|}
\hline \multirow[b]{2}{*}{ Scenario-1 } & \multicolumn{2}{|c|}{ DMDS } & \multicolumn{2}{|c|}{ MMGMT } & \multicolumn{2}{|c|}{ PFEE } & \multicolumn{2}{|c|}{ MADF } \\
\hline & $\begin{array}{c}\text { Average } \\
\text { Y-PSNR } \\
(\mathrm{dB})\end{array}$ & $\begin{array}{c}\text { Y-PSNR } \\
\text { Standard } \\
\text { Deviation }\end{array}$ & $\begin{array}{c}\text { Average } \\
\text { Y-PSNR } \\
\text { (dB) }\end{array}$ & $\begin{array}{c}\text { Y-PSNR } \\
\text { Standard } \\
\text { Deviation }\end{array}$ & $\begin{array}{c}\text { Average } \\
\text { Y-PSNR } \\
(\mathrm{dB})\end{array}$ & $\begin{array}{c}\text { Y-PSNR } \\
\text { Standard } \\
\text { Deviation }\end{array}$ & $\begin{array}{c}\text { Average } \\
\text { Y-PSNR } \\
\text { (dB) }\end{array}$ & $\begin{array}{l}\text { Y-PSNR } \\
\text { Standard } \\
\text { Deviation }\end{array}$ \\
\hline user-1 & 34.4 & 0.8 & 32.5 & 0.8 & 32.6 & 0.7 & 34.1 & 0.5 \\
\hline user-2 & 33.6 & 0.7 & 31.1 & 0.8 & 31.1 & 0.6 & 33.5 & 0.3 \\
\hline user-3 & 33.2 & 0.7 & 30.9 & 0.7 & 30.8 & 0.7 & 33.0 & 0.3 \\
\hline user-4 & 34.9 & 0.9 & 33.0 & 0.9 & 32.9 & 0.8 & 34.5 & 0.6 \\
\hline user-5 & 35.0 & 1.0 & 33.2 & 1.0 & 33.5 & 0.9 & 34.9 & 0.6 \\
\hline \multirow[b]{2}{*}{ Scenario-2 } & \multicolumn{2}{|c|}{ DMDS } & \multicolumn{2}{|c|}{ MMGMT } & \multicolumn{2}{|c|}{ PFEE } & \multicolumn{2}{|c|}{ MADF } \\
\hline & $\begin{array}{c}\text { Average } \\
\text { Y-PSNR } \\
(\mathrm{dB}) \\
\end{array}$ & $\begin{array}{c}\text { Y-PSNR } \\
\text { Standard } \\
\text { Deviation }\end{array}$ & $\begin{array}{c}\text { Average } \\
\text { Y-PSNR } \\
(\mathrm{dB})\end{array}$ & $\begin{array}{c}\text { Y-PSNR } \\
\text { Standard } \\
\text { Deviation }\end{array}$ & $\begin{array}{c}\text { Average } \\
\text { Y-PSNR } \\
(\mathrm{dB}) \\
\end{array}$ & $\begin{array}{c}\text { Y-PSNR } \\
\text { Standard } \\
\text { Deviation }\end{array}$ & $\begin{array}{c}\text { Average } \\
\text { Y-PSNR } \\
(\mathrm{dB}) \\
\end{array}$ & $\begin{array}{c}\text { Y-PSNR } \\
\text { Standard } \\
\text { Deviation }\end{array}$ \\
\hline user-1 & 35.5 & 0.9 & 33.5 & 0.8 & 33.8 & 0.6 & 35.4 & 0.5 \\
\hline user-2 & 35.0 & 0.8 & 32.7 & 0.8 & 33.0 & 0.6 & 34.8 & 0.4 \\
\hline user-3 & 34.5 & 0.78 & 32.3 & 0.7 & 32.9 & 0.7 & 34.4 & 0.4 \\
\hline user-4 & 36.0 & 1.0 & 34.1 & 0.9 & 34.0 & 0.7 & 35.8 & 0.5 \\
\hline user-5 & 36.1 & 1.0 & 34.8 & 1.0 & 34.7 & 0.8 & 36.0 & 0.5 \\
\hline
\end{tabular}

convex function, since for each iteration, $\max \left[z_{l}^{\prime} \sum_{j} M_{s j}^{l} q_{l}\right]$ in (31) does not change with $z_{l}$. Hence, when the proposed scheduling scheme converges to a stable state, the worst distortion reduction is $\sum_{l \in Q^{c}} D\left(z_{l}, z_{l}^{\prime}\right)$.

\section{B. Numerical Results}

In order to provide a meaningful comparison between our proposed MADF approach and other alternative approaches, we consider the use of the recent Max-Min Guaranteed Maximum Throughput (MMGMT) and Proportional Fair Endto-End (PFEE) schemes introduced in [5], and the proposed DMDS scheme in Section III as comparison systems. For each simulation, we generate end-to-end communication streams with random source and destination nodes. 5 users transmit different videos concurrently: user-1 sends video clip City; user-2 sends Mobile; user-3 sends Mother; user-4 sends View; user-5 sends Tennis. The traffic demand for each communication stream for all the schemes is given by a random number uniformly distributed in $\left[0.2 r_{l}^{i n}, 0.6 r_{l}^{i n}\right]$.

We evaluate the performance of the above four schemes in terms of PSNR value under different scenarios. Specifically, Scenario-1 denotes $N=10, L=15, N_{f}=2, N_{n}=2$, and Scenario-2 corresponds to $N=15, L=30, N_{f}=3, N_{n}=3$. The simulation results are presented in Table IV. It should be noted that all the simulation results in this subsection have been obtained using 300 runs in order to obtain statistically meaningful average values. Based on the given objective simulation results, there are two main observations:

- With regards to the average PSNR value, MADF achieves a satisfying performance. Its performance is comparable to that of DMDS, and much better than those of MMGMT and PFEE schemes. For example, the average performance differences between MADF and DMDS for 5 users in two scenarios are only $0.2 \mathrm{~dB}$ and $0.1 \mathrm{~dB}$, respectively. However, compared to MMGMT and PFEE schemes, the proposed MADF scheme can achieve $1.7 \mathrm{~dB}$ and $1.7 \mathrm{~dB}$ performance improvement for scenario-1, and $1.9 \mathrm{~dB}$ and $1.8 \mathrm{~dB}$ for scenario-2. That is to say, MADF is derived from DMDS, and holds the basic characteristics of DMDS.
- With regards to the PSNR derivation value, MADF has the best constant performance. We can observe that although DMDS scheme globally achieves the best PSNR performance, it results in a severe unfairness on performance. In addition, MMGMT and PFEE schemes also consider the fairness problems in scheduling, and they can achieve certain performance improvement compared to DMDS scheme. However, MMGMT and PFEE schemes do not consider the content of video, as expected, their performances are worse than the proposed MADF scheme.

\section{CONCLUSions}

In this paper, we have developed fully distributed scheduling schemes that jointly solve the channel-assignment, rate allocation, routing and fairness problems for video streaming over multi-channel multi-radio networks. Unlike conventional scheduling schemes focus on optimal system throughput or scheduling efficiency, our work aims at achieving minimal video distortion and certain fairness by jointly considering media-aware distribution and network resource allocation. Extensive simulation results are provided which demonstrate the effectiveness of our proposed schemes.

The results in this paper have some interesting implications on the practical use of multi-radio multi-channel multi-hop wireless networks, i.e., multimedia sensor network is a good example. As we know, current sensor networks due to their limit transmit capacities can hardly transmit large amount of multimedia data concurrently. Multi-channel multi-radio technique is a direction to provide a satisfying multimedia service in wireless sensor networks. In addition, 3GPP LTE (Long Term Evolution) system using relay is also an example. As we know, LTE uses OFDM (Orthogonal Frequency Division Multiplexing) for the downlink, in which the transmitter sends information over a large number of sub-carriers [2]. So it can be viewed as a special type of multi-channel multi-radio multi-hop wireless system. Therefore, our proposed video scheduling scheme for multi-channel multi-radio multi-hop wireless networks is eager to have large application ground.

For future work, we plan to study some practical issues for implementing the proposed schemes. Note that in real 
video transmission over multi-channel multi-radio systems, additional works need to be developed in order to: i) reduce the dependence of the video content for scheduling scheme can automatically adapt; ii) simplify the scheduling scheme, especially the channel and link information exchange and feedback; iii) extend the results to more practical systems (e.g., OFDM) and channel models (e.g., heterogeneous channels). In our ongoing work, we plan to carefully address these open problems and study their impacts on the actual multi-channel multi-radio systems.

\section{ACKNOWLEDGEMENT}

This work is supported by National Fundamental research grant (2010CB731803, 2009CB3020402), NSF China (No. 60702046, 60832005, 60632040); China Ministry of Education (No. 20070248095); Qualcomm Research Grant; China International Science and Technology Cooperation Programm (No. 2008DFA11630); PUJIANG Talents (08PJ14067); Shanghai Innovation Key Project (08511500400); National High tech grant (2007AA01Z267, 2009AA01Z248). Moreover, the authors also thank the anonymous reviewers for insightful comments and suggestions.

\section{APPENDIX}

Proof of Proposition 1: Since (15) is a convex optimization problem satisfying Slater's condition, the duality gap is zero. Therefore, a distributed algorithm for (15) can be derived through the Lagrange dual problem. First we form the following Lagrangian:

$$
L\left(D_{\text {all }}, \mathcal{M}, \phi_{l}\right)=\sum_{s} \sum_{j} D_{\text {all }}-\sum_{l} \phi_{l}(t)\left(r_{l}^{\text {in }}-r_{l}^{\text {out }}\right) .
$$

However, (34) can not be decoupled yet because $\phi_{l}$ refers to many variables. Therefore, we keep on introducing a new variable $\kappa_{s}$ and additional constraints $P T A_{l}^{\prime}$ and $\triangle R_{s}^{k}$. Note that $P_{s}^{k}$ corresponds to the path $j$ of user $s$, so

$$
\begin{aligned}
L\left(D_{\text {all }}, \mathcal{M}, \phi_{l}, \kappa_{s}\right) & =\sum_{s} \kappa_{s}(t) D_{\text {all }}-\sum_{l} \phi_{l}(t)\left(r_{l}^{\text {in }}-r_{l}^{\text {out }}\right) \\
& +\sum_{l \in P_{s}^{k}} \kappa_{s}(t) \frac{\triangle R_{s}^{k}(t)}{P T A_{l}^{\prime}} .
\end{aligned}
$$

So far, (35) can be decoupled with three sub-problems as follows:

- Each source $s$ :

$$
\max _{F_{s}}-\sum_{j} \kappa_{s}(t)\left(F_{s j}\right)^{2}-\sum_{j} F_{s j} \sum_{l} \phi_{l}(t) M_{s j}^{l} q_{l}(t)
$$

where $q_{l}(t)$ is the queue length of link $l$ at time slot $t$.

- Each link $l$ :

$$
\min _{P_{s}^{k}} \sum_{l \in P_{s}^{k}} \kappa_{s}(t) \frac{\triangle R_{s}^{k}(t)}{P T A_{l}^{\prime}} .
$$

- Each channel $c$ :

$$
\min \sum_{l} \phi_{l}(t)\left(r_{l}^{\text {in }}-r_{l}^{\text {out }}\right) .
$$

The Lagrangian dual function $L_{d}(\phi, \kappa)$ is defined as the maximized $L\left(D_{\text {all }}, \mathcal{M}, \phi, \kappa\right)$ over $D_{\text {all }}$ and $\mathcal{M}$ for given $\phi$ and $\kappa$. Each source can compute an optimizer $D_{\text {all }}^{*}$ and each link $l$ and channel $c$ can compute an optimizer $\mathcal{M}^{*}(\phi, \kappa)$. The Lagrange dual problem of (15) is:

$$
\min L_{d}\left(\phi_{l}, \kappa_{s}\right)=L\left(D_{\text {all }}^{*}, \mathcal{M}^{*}\left(\phi_{l}, \kappa_{s}\right), \phi_{l}, \kappa_{s}\right),
$$

where $\left(\phi_{l}, \kappa_{s}\right)$ are the dual variables. Note that (39) is a convex minimization. In addition, we can define the iteration method for $u_{l}^{c}(t)$ as:

$$
u_{l}^{c}(t+1)=u_{l}(t+1) / r_{l}^{c}(t+1) .
$$

Since $L_{d}\left(\phi_{l}, \kappa_{s}\right)$ may be non-differentiable, an iterative subgradient method can be used to update the dual variables to solve (39).

- Queue Length Update:

$q_{l}(t+1)=\left[q_{l}(t)+\lambda_{q}(t)\left(\sum_{s} \sum_{j} M_{s j}^{l} F_{s j} R_{s}^{j}(t)-\sum_{c} r_{l}^{c}(t)\right)\right]^{+}$,

where $\lambda_{q}(t)$ represents the queue length step size.

- Rate Increment Update:

$\triangle R_{s}^{k}(t+1)=\left[\triangle R_{s}^{k}(t)+\lambda_{R}(t)\left(\sum_{k} \triangle D_{\text {loss }}^{k}-\triangle D_{\text {comp }}^{k}\right)\right]^{+}$,

where $\lambda_{R}(t)$ represents the rate increment step size.

(41) and (42) are exactly the DMDS scheme steps described in Table II. Certain choices of step sizes, such as $\lambda_{q}(t)=$ $\lambda_{1} / t, \lambda_{R}(t)=\lambda_{2} / t$ where $\lambda_{1}>0, \lambda_{2}>0$, guarantee that this algorithm will converge to the joint optimum. In this case, the convergent point is a globally optimal $\mathcal{M}$ to the problem (15) since we have shown that the problem can be written as convex optimization.

\section{REFERENCES}

[1] M. Alicherry, R. Bathia, and L. Li, "Joint Channel Assignment and Routing for Throughput Optimization in Multi-Radio Wireless Mesh Networks," IEEE J. Sel. Areas Commun., vol. 24, no. 11, pp. 1960-1971, 2006.

[2] X. Lin and S. Rasool, "A Distributed Joint Channel-Assignment, Scheduling and Routing Algorithm for Multi-Channel Ad Hoc Wireless Networks, " in Proc. of IEEE INFOCOM, 2007.

[3] M. Kodialam and T. Nandagopal, "Characterizing the Capacity Region in Multi-Radio Multi-Channel Wireless Networks", in Proc. of ACM MobiCom, 2005.

[4] A. Raniwala and T. Chiueh, "Architecture and Algorithms for an IEEE 802.11-Based Multi-Channel Wireless Mesh Network," in Proc. of IEEE INFOCOM, 2005.

[5] J. Tang, G. Xue and W. Zhang, "Cross-layer Design for End-to-End Throughput and Fairness Enhancement in Multi-Channel Wireless Mesh Networks," IEEE Trans. Wireless Commun., vol. 6, no. 10, pp. 3482-3486, 2007.

[6] L. Chen, Q. Zhang, and M. Li, "Joint Topology Control and Routing in Multi-radio Multi-channel Wireless Mesh Networks," IEEE Trans. Veh. Technol., vol. 56, no. 5, pp. 1101-1112, 2007.

[7] J. Tang, G. Xue and W. Zhang, "Interference-Aware Topology Control and QoS Routing in Multi-Channel Wireless Mesh Networks," in Proc. ACM MobiHoc, 2005.

[8] H. Wu, F. Yang, K. Tan, J. Chen, Q. Zhang, and Z. Zhang, "Distributed Channel Assignment and Routing in Multi-radio Multi-channel Multihop Wireless Networks", IEEE J. Sel. Areas Commun., vol. 24, no. 11, pp. 1972-1983, 2006.

[9] L. Zhou, B. Geller, B. Zheng, A. Wei, and J. Cui, "Distributed Resource Allocation for Multi-Source Multi-Description Multi-Path Video Streaming over Wireless Networks," IEEE Trans. Broadcast, vol. 55, no. 4, pp. 731-741, Dec. 2009.

[10] L. Zhou, B. Geller, A. Wei, and B. Zheng, "Cross-Layer Rate Allocation for Multimedia Applications in Pervasive Computing Environment," in Proc. of IEEE GLOBECOM, 2008. 
[11] D. Jurca, W. Kellerer, E. Steinbach, S. Khan, S. Thakolsri, P. Frossard, "Joint Network and Rate Allocation for Video Streaming over Multiple Wireless Networks," in Proc. of IEEE ISM, 2007.

[12] Q. Zhang and Y. Zhang, "Cross-Layer Design for QoS Support in Multihop Wireless Networks," Proc. IEEE, vol. 96, no. 1, pp. 64-76, 2008.

[13] E. Setton, T. Yoo, X. Zhu, A. Goldsmith and B. Girod, "Cross-layer Design of Ad Hoc Networks for Real-Time Video Streaming," IEEE Wireless Commun. Mag., vol. 12, no. 4, pp. 59-65, 2005.

[14] D. Jurca and P. Frossard, "Packet Selection and Scheduling for Multipath Video Streaming," IEEE Trans. Multimedia, vol. 9, no. 3, pp. 629-641, 2007.

[15] W. Wang, M. Palaniswami, and S. H. Low, "Application-Oriented Flow Control: Fundamentals, Algorithms and Fairness," IEEE/ACM Trans. Netw., vol. 14, no. 6, pp. 1282-1291, 2006.

[16] T. Ozcelebi, M. O. Sunay, M. R. Civanlar, and A. M. Tekalp, "Application QoS Fairness in Wireless Video Scheduling," in Proc. of IEEE SPCA, 2006

[17] R. Braden, D. Clark, and S. Shenker, "Integrated Services in the Internet Architecture: An Overview," RFC 1633, 1994.

[18] S. Blake, D. Black, M. Carlson, E. Davies, Z. Wang, and W. Weiss, "An Architecture for Differentiated Services," $R F C$ 2475, 1998.

[19] X. Zhu, E. Setton, and B. Girod, "Congestion-distortionoptimized video transmissionoveradhocnetworks," SignalProcess: Image Communications, vol. 20, no. 8, pp. 77-83, 2005.

[20] S. Pack, X. Shen, J. W. Mark and L. Cai, "A Two-Phase Loss Differentiation Algorithm for Improving TFRC Performance in IEEE 802.11 WLANs," IEEE Trans. Wireless Commun., vol. 6, no. 11, pp. 4164-4175, 2007.

[21] K. Stuhlmuller, N. Farber, M. Link and B. Girod, "Analysis of Video Transmission over Lossy Channels", IEEE J. Sel. Areas Commun., vol. 18, no. 6, pp. 1012-1032, 2000.

[22] X. Zhu, E. Setton and B. Girod, "Congestion-Distortion Optimized Video Transmission over Ad Hoc Networks", J. Signal Processing: Image Communication, vol. 20, pp. 773-783, 2005.

[23] Q. Qu, Y. Pei and J. W. Modestino, "An Adaptive Motion-Based Unequal Error Protection Approach For Real-Time Video Transport over Wireless IP Networks," IEEE Trans. Multimedia, vol. 8, no. 5, pp. 1033-1044, 2006.

[24] L. Kleinrock, Queuing Systems, Volume II: Computer Applications, Wiley Interscience, New York, USA, 1976.

[25] Y. Li, Z. Li, M. Chiang, and A. R. Calderbank, "Content aware distortion fair video streaming in networks," IEEE Trans. Multimedia, vol. 11, no. 6, pp. 1182-1193, 2009.

[26] H. Park and M. Schaar, "Fairness Strategies for Multi-User Multimedia Applications in Competitive Environments using the Kalai-Smorodinsky Bargaining Solution," in Proc. of IEEE ICASSP, 2007.

[27] J. W. Lee, M. Chiang, and R. A. Calderbank, "Price-based distributed algorithm for optimal rate-reliability tradeoff in network utility maximization," IEEE J. Sel. Areas Commun., vol. 24, no. 5, pp. 962-976, 2006.

[28] Y. Li, M. Chiang, A. R. Calderbank, S. Diggavi, "Optimal ratereliability-delay tradeoff in networks with composite links," IEEE Trans. Commun.,vol. 57, no. 5, pp. 1390-1401, May 2009.

[29] J. Tang and X. Zhang, "Cross-Layer Resource Allocation Over Wireless Relay Networks for Quality of Service Provisioning," IEEE J. Sel. Areas Commun., vol. 25, no. 4, pp. 645-657, 2007.

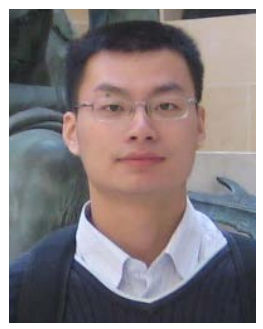

Liang Zhou received his B.S. degree and M.S. degree (with honors) both major at Electronic Engineering from Nanjing University of Posts and Telecommunications, Nanjing, China in 2003 and 2006, respectively. In March 2009, he received his $\mathrm{Ph} . \mathrm{D}$. degree major at Electronic Engineering both from Ecole Normale Superieure (E.N.S.), Cachan, France and Shanghai Jiao Tong University, Shanghai, China. From Mar. 2009, he is a postdoctoral researcher in ENSTA-ParisTech, Paris, France. His research interests are in the area of multimedia communications and services, in particular, resource allocation, scheduling, and cross-layer design.

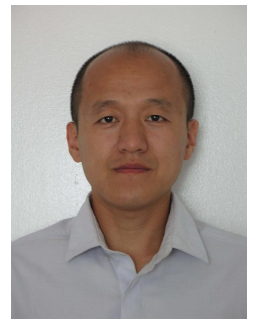

Xinbing Wang received the B.S. degree (with hons.) from the Department of Automation, Shanghai Jiao Tong University, Shanghai, China, in 1998, and the M.S. degree from the Department of Computer Science and Technology, Tsinghua University, Beijing, China, in 2001. He received the Ph.D. degree, major in the Department of electrical and Computer Engineering, minor in the Department of Mathematics, North Carolina State University, Raleigh, in 2006. Currently, he is an associate Professor in the Department of Electronic Engineering, Shanghai Jiao Tong University, Shanghai, China. His research interests include resource allocation and management in mobile and wireless networks, TCP asymptotics analysis, wireless capacity, cross layer call admission control, asymptotics analysis of hybrid systems, and congestion control over wireless ad hoc and sensor networks. Dr. Wang has been a member of the Technical Program Committees of several conferences including IEEE INFOCOM 2009-2011, IEEE ICC 2007-2011, IEEE Globecom 2007-2010.

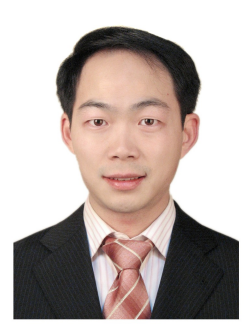

Wei Tu received the B.S. from East China University of Science \& Technology, China, in 1999 and the M.S. degree in electronic engineering from Technische Universität München, Germany, in 2003. From 2004-2008 he was a member of the research staff of the Media Technology Group at Technische Universität München, where he researched on proxy-based video transmission related topics and obtained his Ph.D degree in 2009. After that, he joined Nokia Siemens Networks, engaged in network and service optimization for multimedia

applications in $3 \mathrm{G}$ networks.

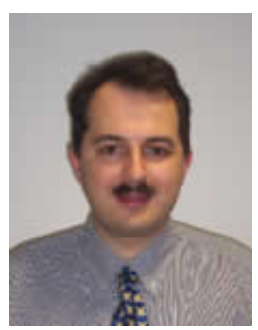

Gabriel-Miro Muntean is a Lecturer with the School of Electronic Engineering, Dublin City University, Ireland, where he obtained his Ph.D. degree in 2003 for research in quality-oriented adaptive multimedia streaming over wired networks. He was awarded the B.Eng. and M.Sc. degrees in Software Engineering from the Computer Science Department, "Politehnica" University of Timisoara, Romania in 1996 and 1997 respectively. Dr. Munteans research interests include QoS and performance issues of adaptive multimedia streaming, and personalised eLearning over wired and wireless networks and with various devices. He has authored a book and edited two more, along with four book chapters and has published many papers in prestigious international conferences and journals.

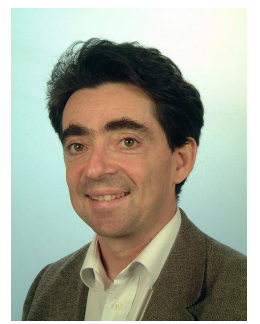

Benoit Geller received the Master Sc. in Electrical Engineering from the ENSERB in 1988, the Ph.D. in Telecommunications from the INPG in 1992, and the Accreditation to Supervise Research HDR in Information Sciences from the University of Paris 12 in 2004. He was the head of the Multisensor and Information Team (TIM, 17 permanent researchers) at SATIE lab-Ecole Normale Superieure de Cachan until he joined ENSTA ParisTech in 2007-08 where is currently a Professor in telecommunications. He is working on iterative methods with application to telecommunication networks. He also has a strong experience of Digital Communications Systems with emphasis on Mobile and Broadband Systems, Information theory and Channel Coding, Signal Processing applied to telecommunications. He has been involved in several European projects (Mast AIDA, Medea INCA, Medea MIDAS, FP6 Newcom) and has also been in charge of many industrial projects. He has published about 50 international papers and has pended 4 international patents. 